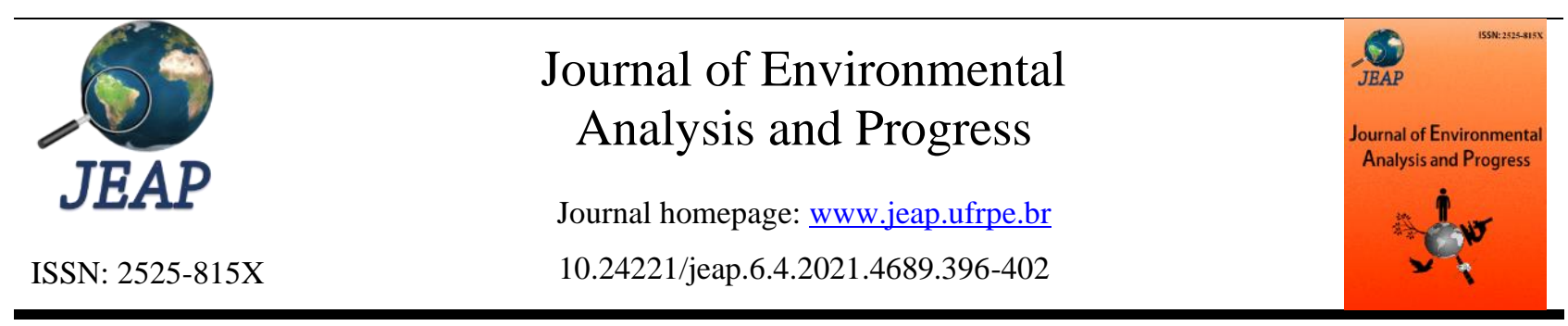

\title{
Reação de acessos de Cenchrus a Pyricularia grisea no semiárido brasileiro
}

\section{Reaction of Cenchrus accessions to Pyricularia grisea in the semiarid region of Brazil}

\begin{abstract}
Rafaela Priscila Antonio ${ }^{\mathrm{a}}$, Pedro Martins Ribeiro Júnior ${ }^{\mathrm{a}}$, Irlane Cristine de Souza Andrade Lira ${ }^{\mathrm{b}}$, Lucas Peixinho Campos Nery ${ }^{a}$, Jullie Cryscelle Leandro Silva ${ }^{c}$, Bruno Djvan Ramos Barbosa ${ }^{a}$, Breno dos Santos Costa ${ }^{\mathrm{c}}$

a Empresa Brasileira de Pesquisa Agropecuária-Embrapa Semiárido, BR 428, Km 152, Zona Rural - Caixa Postal 23, Petrolina, Pernambuco, Brasil. CEP: 56302-970. E-mail: rafaela.antonio@embrapa.br, pedro.ribeiro@embrapa.br, lucaspeixinho17@gmail.com, brunodj31@hotmail.com.

b Universidade Estadual de Feira de Santana-UEFS, Programa de Pós-graduação em Recursos Genéticos Vegetais. Avenida Transnordestina, s/n, Novo Horizonte, Feira de Santana, Bahia, Brasil. CEP: 44036-900. E-mail: irlane.cristine@gmail.com.

${ }^{\mathrm{c}}$ Universidade de Pernambuco-UPE, Campus Petrolina, BR 203, Km 2, s/n, Vila Eduardo, Petrolina, Pernambuco, Brasil. CEP: 56328-900. E-mail: julliecryscelle@gmail.com, brenocost27@gmail.com.
\end{abstract}

\section{A R T I C L E I N F O}

Recebido 08 Out 2021

Aceito 20 Nov 2021

Publicado 09 Dez 2021

\begin{abstract}
A B S T R A C T
The fungus Pyricularia grisea causes leaf spots on buffel grass (Cenchrus ciliaris). This fungus is a major problem in these forage pastures in Texas, USA, and Mexico. Although this disease does not present serious problems to buffelgrass in the Brazilian semiarid region, the search for germplasm with some level of tolerance to this disease is always important before they cause problems for the crop. This study aimed to evaluate the reaction of the Cenchrus spp. to the $P$. grisea, in natural infection. Twenty accessions belonging to the Cenchrus Active Germplasm Bank (AGB) from Embrapa Semiárido were evaluated in randomized blocks with three replications and a plot containing 20 plants. The evaluations were carried out in two evaluation cycles in 2019 and 2020, in the rainy season, when the environmental conditions for the development of the disease were favorable. The severity of the disease was assessed through a grading scale ranging from 1 to 4 , and the percentage of injured leaf areas was used. The average data of the scores per plot were submitted to analysis of variance, and the averages were compared by the Scott and Knott test, at $5 \%$ significance. There was a significant effect on disease tolerance. Ten accesses received an average score above 3 (CPATSA 83476; CPATSA 79151; CPATSA 79150; CPATSA 79591; CPATSA 90194; CPATSA 79144; CPATSA 79558; CPATSA 79134; CPATSA 90570; CPATSA 79613), in both assessments, indicating a moderate resistance concerning the susceptible witness, the Biloela cultivar. There is variability to be explored for the reaction to P. grisea in the Cenchrus AGB from Embrapa Semiárido.
\end{abstract}

Keywords: Magnaporthe grisea, buffel grass, genetic resistance.

\section{R E S U M O}

O fungo Pyricularia grisea causa manchas foliares em capim buffel (Cenchrus ciliaris), sendo um problema em pastagens dessa forragem no Texas, EUA e México. Apesar desta doença não apresentar problemas graves ao capim buffel no Semiárido brasileiro, a busca de germoplasma com algum nível de tolerância a esta doença tem importância preventiva. O objetivo neste estudo foi avaliar a reação de acessos de Cenchrus spp. a $P$. grisea, em infecção natural. Foram avaliados 20 acessos pertencentes ao Banco Ativo de Germoplasma (BAG) de Cenchrus da Embrapa Semiárido, em blocos casualizados com três repetições e parcela contento 20 plantas. As avaliações foram realizadas em dois ciclos de avaliação em 2019 e 2020, no 
período chuvoso, quando as condições ambientais para o desenvolvimento da doença eram favoráveis. Para a avaliação da severidade da doença foi utilizada uma escala de notas variando de 1 a 4 e percentual da área foliar lesionada. Os dados médios das notas por parcelas foram submetidos a análise de variância e as médias comparadas pelo teste de Scott e Knott, à 5\% de significância. Houve efeito significativo para a tolerância à doença. Dez acessos receberam nota média acima de 3 (CPATSA 83476; CPATSA 79151; CPATSA 79150; CPATSA 79591; CPATSA 90194; CPATSA 79144; CPATSA 79558; CPATSA 79134; CPATSA 90570; CPATSA 79613), nas duas avaliações, indicando uma moderada resistência em relação a testemunha suscetível, a cultivar Biloela. Existe variabilidade a ser explorada para reação a $P$. grisea no BAG de Cenchrus da Embrapa Semiárido.

Palavras-Chave: Magnaporthe grisea, capim buffel, resistência genética.

\section{Introdução}

O capim buffel (Cenchrus ciliaris L.) é uma planta perene, nativa da África, Ásia e de regiões do Oriente Médio (Tix, 2000), foi introduzido como forrageira em terras áridas das Américas e da Austrália nos séculos 19 e 20 (Marshall et al., 2012).

No Brasil, esta planta foi introduzida na década de 1950, especificamente em São Paulo, de onde foi, posteriormente, distribuída para o nordeste, após ter passado por algumas avaliações iniciais (Oliveira, 2005). Apresentou grande sucesso nas regiões Semiáridas do Brasil, como também do mundo, devido à sua resistência ao pastoreio pesado e a longos períodos de estiagem (Sousa \& Araújo Filho, 2007; Farrell \& Gornish, 2019), consequência do seu sistema radicular profundo que consegue chegar aos perfis mais profundos do solo, proporcionando maior contato com a água e nutrientes.

O capim buffel tem a capacidade de adaptação a solos de textura leve a pesada, de acidez moderada, com fertilidade média, sendo exigente principalmente em fósforo. A temperatura ótima para a fotossíntese é de $35^{\circ} \mathrm{C}$, não sendo tolerante ao frio e ao encharcamento do solo. Requer precipitações entre 300 e $800 \mathrm{~mm}$, no entanto, pode sobreviver em áreas com precipitação em torno de $100 \mathrm{~mm}$ (Oliveira, 2005; Sousa \& Araújo Filho, 2007; Moreira et al., 2007; Miller et al., 2010; Mnif \& Chaieb, 2010).

Diversas pragas e doenças podem causar problemas no desenvolvimento e crescimento do capim buffel, o que pode afetar a produção de biomassa, a qualidade da pastagem e a produção de sementes desta gramínea. Uma destas doenças é a mancha foliar causada pelo fungo Pyricularia grisea (Cooke) Sacc., cuja forma teleomórfica é Magnaporthe grisea (Hebert) Barr (Bedendo \& Prabhu, 2016). Esta espécie de fungo pode infectar mais de 50 espécies de plantas, incluindo arroz (Oryza sativa), trigo (Triticum aestivum), cevada (Hordeum vulgare), painço (Setaria itálica), milheto (Panicum miliaceum), capim-pé-degalinha (Eleusine coracana), azevém perene
(Lolium perenne), capim-colchão (Digitaria sanguinalis) e capim angola (Brachiaria mutica) (Madhavan et al., 2014).

Em capim buffel, $P$. grisea foi relatado pela primeira na Austrália, em 1998 (Perrot \& Chakraborty, 1999). Além da Austrália, é um grande problema em pastagens em regiões produtoras dos EUA, como o sul do Texas e o norte do México (Rodriguez et al., 1999). O fungo causa, inicialmente, manchas escuras e descoloridas na folha que se desenvolvem em lesões necróticas arredondadas a elípticas com uma borda vermelha escura e um halo clorótico amarelo, em situações de infecções severas, as lesões coalescem, necrosando toda a lâmina foliar (Rodriguez et al., 1999).

A mancha foliar pode reduzir no capim buffel a quantidade e a qualidade da folhagem e a quantidade de sementes produzidas (Flores et al., 2019), devido a diminuição da área foliar capaz de realizar a fotossíntese. Sob estresse de calor e umidade, as folhas com poucas lesões e descoloração amarela murcham completamente, que se assemelha a murchas causadas por estresse hídrico ou lesão por herbicida. O patógeno também reduz a quantidade e qualidade da semente ao infectar o envoltório da cabeça (Rodriguez et al., 1999). Estudos sobre os aspectos da epidemia de $P$. grisea em Cenchrus spp. é limitado. Em outras gramíneas, temperaturas ótimas para infecção por $P$. grisea variaram de $25^{\circ} \mathrm{C}$ a $29^{\circ} \mathrm{C}$ e, em temperaturas abaixo de $5^{\circ} \mathrm{C}$ ou acima de $35^{\circ} \mathrm{C}$ não foram observados sintomas. Rodríguez et al. (1999) observaram que o desenvolvimento da doença no buffel em campo indicaram que a maior severidade da doença foi associada com uma umidade relativa maior que $75 \%$ e noites com 8-10 $\mathrm{h}$ de duração. $P$. grisea sobrevive em resíduos vegetais infectados e gramíneas espontâneas, que podem atuar como fonte de inóculo primário para o posterior desenvolvimento da doença.

O capim buffel se reproduz, majoritariamente, por apomixia, que é a formação de sementes sem fertilização sexual. Desta forma, a progênie é geneticamente idêntica à planta mãe, 
o que produz baixa diversidade genética em pastagens. $\mathrm{O}$ aumento das pastagens cultivadas de capim buffel no semiárido, aliado ao baixo número de cultivares atualmente em uso associado às condições meteorológicas favoráveis, principalmente na época chuvosa, pode ocorrer o favorecimento da disseminação do fungo (Rodriguez et al., 1999).

O objetivo do estudo foi avaliar a reação de 20 acessos do Banco Ativo de Germoplasma de Cenchrus da Embrapa Semiárido à $P$. grisea em infecção natural no campo.

\section{Material e Métodos}

Foram avaliados 20 acessos do gênero Cenchrus oriundos do Banco de Germoplasma de Cenchrus da Embrapa Semiárido. Desses, 18 acessos: CPATSA 7757; CPATSA 79129; CPATSA 79144; CPATSA 79148; CPATSA 79147; CPATSA 79149; CPATSA 79150; CPATSA 79151; CPATSA 80194; CPATSA 80199; CPATSA 80200; CPATSA 83438; CPATSA 83476; CPATSA 90558; CPATSA 90570; CPATSA 90591; CPATSA 90592 e CPATSA 90613, e duas testemunhas: CPATSA 7602 (cultivar Biloela) e CPATSA 79123 (cultivar West Australian).

$\mathrm{O}$ ensaio foi realizado em Campo Experimental da Caatinga da Embrapa Semiárido nos anos de 2019 e 2020, em Petrolina, Pernambuco. O delineamento utilizado foi o de blocos ao acaso com 20 tratamentos (18 acessos + duas cultivares), com três repetições com parcelas medindo $6 \mathrm{~m}^{2}$, em condições de sequeiro. A densidade de plantio utilizada foi de 33 mil plantas.ha ${ }^{-1}$, com espaçamento de $1,0 \mathrm{~m}$ entre linhas e $0,3 \mathrm{~m}$ entre plantas. A semeadura do experimento foi realizada em janeiro de 2019. Para a adubação do plantio foram utilizados $40 \mathrm{~kg} \cdot \mathrm{ha}^{-1}$ de nitrogênio, 100 kg.ha- ${ }^{-1}$ de $\mathrm{P}_{2} \mathrm{O}_{5}, 120$ kg.ha- ${ }^{-1}$ de $\mathrm{K}_{2} \mathrm{O}$ e 10 t.ha $^{-1}$ de esterco caprino.

Para a avaliação da reação dos genótipos ao fungo $P$. grisea foi utilizada uma escala de notas variando de 1 a 4, em duas avaliações realizadas no período chuvoso, em abril de 2019 e março de 2020, onde: 1 - Sintomas de manchas nas folhas e colmo no terço superior das plantas (susceptível); 2 - Sintomas da doença até o terço médio das plantas (moderadamente suscetível); 3 - Sintomas da doença apenas no terço inferior das plantas (moderadamente resistente); 4 - Sem sintomas visíveis da doença (resistente).

$\mathrm{Na}$ última avaliação foi realizada também, para a confirmação dos resultados, uma avaliação da severidade da doença coletando-se 10 folhas do terço médio das plantas da parcela de forma aleatória, para quantificação da percentagem da área foliar lesionada pela doença.

As médias das notas da reação dos genótipos e da severidade da doença foram submetidas ao teste $\mathrm{F}$ e agrupadas utilizando-se o teste de Scott \& Knott $(\mathrm{p}<0,05)$, por meio do software Sisvar (Ferreira, 2011).

\section{Resultados e Discussão}

Manchas foliares causadas por $P$. grisea foi a doença que predominou no campo no período avaliado (Figura 1).

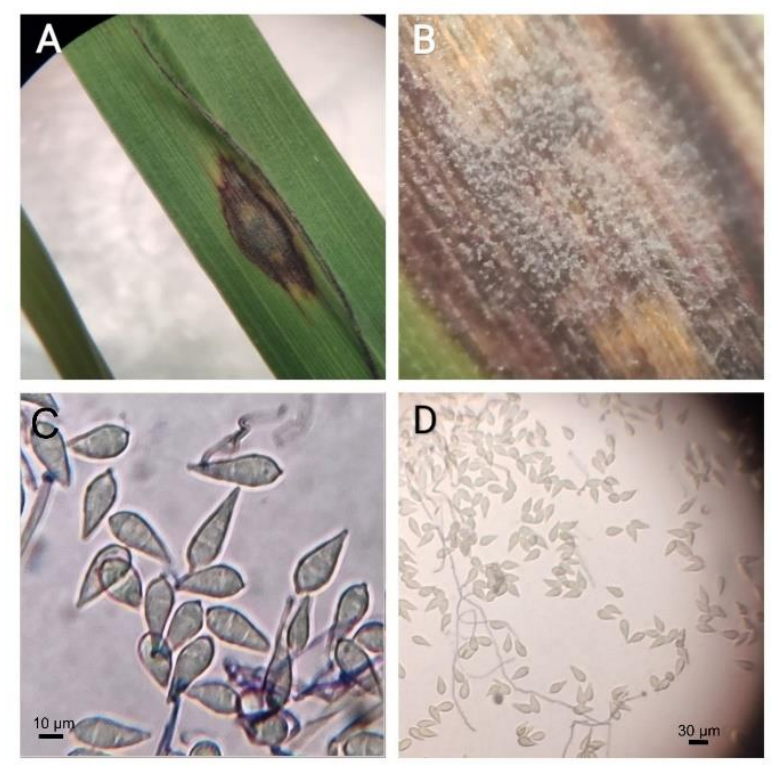

Figura 1. Folha de capim buffel (Cenchrus ciliaris) A. Sintoma foliar observado no experimento causado por Pyricularia grisea; B. produção de estruturas de reprodução; C-D. conídios produzidos nas lesões. Fonte: Antonio et al. (2021).

Nos primeiros meses de 2020, janeiro a abril, as condições foram mais favoráveis ao fungo do que o mesmo período de 2019, pois as plantas apresentaram em média nota de 2,1 , enquanto em 2019, essa nota foi de 2,7 (Tabela 1).

Tabela 1. Reação de genótipos de capim buffel (Cenchrus ciliaris) à mancha foliar causada por Pyricularia grisea em campo sob infecção natural em Petrolina, Pernambuco Fonte: Antonio et al. (2021).

\begin{tabular}{ccccc}
\hline \multirow{2}{*}{ Genótipo } & \multicolumn{4}{c}{ Notas de avaliação } \\
\cline { 2 - 6 } & Abril 2019 & Março 2020 \\
\hline CPATSA 83476 & 3,0 & b & 3,7 & $\mathrm{C}$ \\
CPATSA 79151 & 3,3 & $\mathrm{~b}$ & 3,3 & $\mathrm{C}$ \\
CPATSA 79150 & 3,7 & $\mathrm{~b}$ & 3,3 & $\mathrm{C}$ \\
CPATSA 79591 & 2,7 & $\mathrm{a}$ & 3,3 & $\mathrm{C}$ \\
CPATSA 90194 & 3,3 & $\mathrm{~b}$ & 3,0 & $\mathrm{C}$ \\
CPATSA 79144 & 4,0 & $\mathrm{~b}$ & 3,0 & $\mathrm{C}$ \\
CPATSA 79148 & 2,3 & $\mathrm{a}$ & 3,0 & $\mathrm{C}$ \\
CPATSA 79558 & 3,0 & $\mathrm{~b}$ & 3,0 & $\mathrm{C}$ \\
\hline
\end{tabular}




\begin{tabular}{cllll}
\hline CPATSA 79134 & 4,0 & $\mathrm{~b}$ & 3,0 & $\mathrm{C}$ \\
CPATSA 90570 & 3,7 & $\mathrm{~b}$ & 2,0 & $\mathrm{~B}$ \\
CPATSA 79613 & 4,0 & $\mathrm{~b}$ & 1,7 & $\mathrm{~B}$ \\
CPATSA 90592 & 2,3 & $\mathrm{a}$ & 1,7 & $\mathrm{~B}$ \\
CPATSA 80200 & 2,0 & $\mathrm{a}$ & 1,0 & $\mathrm{~A}$ \\
CPATSA 80199 & 3,0 & $\mathrm{~b}$ & 1,0 & $\mathrm{~A}$ \\
BILOELA & 2,0 & $\mathrm{a}$ & 1,0 & $\mathrm{~A}$ \\
CPATSA 7757 & 2,0 & $\mathrm{a}$ & 1,0 & $\mathrm{~A}$ \\
CPATSA 79129 & 1,3 & $\mathrm{a}$ & 1,0 & $\mathrm{~A}$ \\
CPATSA 79149 & 1,3 & $\mathrm{a}$ & 1,0 & $\mathrm{~A}$ \\
CPATSA 79147 & 2,0 & $\mathrm{a}$ & 1,0 & $\mathrm{~A}$ \\
West Australian & 1,0 & $\mathrm{a}$ & 1,0 & $\mathrm{~A}$ \\
\hline Média & 2,7 & & 2,1 & \\
\hline
\end{tabular}

Médias com mesma letra na coluna não diferem pelo teste de Scott \& Knott $(\mathrm{p}<0,05)$. Notas de avaliação da reação dos genótipos ao fungo Pyricularia grisea: $1=$ Sintomas de manchas nas folhas e colmo no terço superior das plantas (susceptível); 2 = Sintomas da doença até o teço médio das plantas (moderadamente suscetível); $3=$ sintomas da doença apenas no terço inferior das plantas (moderadamente resistente); $4=$ sem sintomas da doença (resistente).

$\mathrm{Na}$ escala de notas utilizada para a avaliação da reação dos genótipos ao fungo, quanto mais próximo da nota 1 , maior a severidade da doença. A maior intensidade da doença em 2020 ocorreu, provavelmente, pela maior precipitação, maior umidade relativa e menor temperatura média em relação ao ano de 2019 nos primeiros meses do ano (Figura 2).

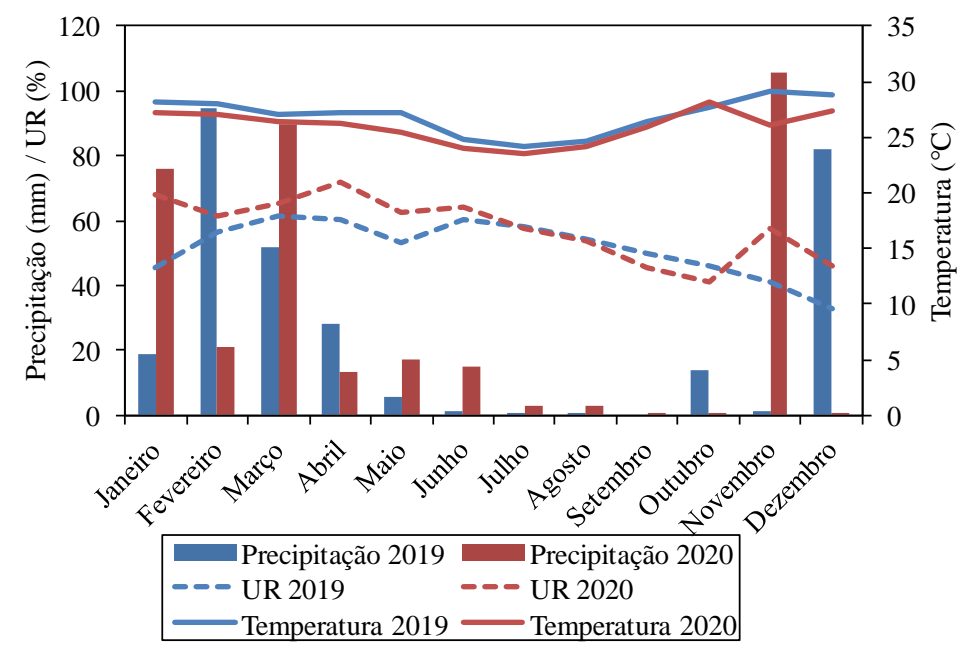

Figura 2. Médias climatológicas de temperatura, umidade relativa (UR) e precipitação (mm) no município de Petrolina-PE, nos anos de 2019 e 2020. Fonte: http://labmet.univasf.edu.br/joomla/index.php/dadosclimaticos.

Foi observada variabilidade na reação dos acessos do BAG de Cenchrus a $P$. grisea. As cultivares usadas como testemunhas nesse ensaio experimental foram consideradas suscetíveis. A Biloela obteve nota média igual a 1,5 sendo considerada suscetível como relatado na literatura por Perrott \& Chakraborty (1999). A cultivar West Australian apresentou baixa tolerância ao fungo, com nota média de 1,0, caracterizando também como suscetível.

Nas avaliações realizadas, pelo menos nove acessos, CPATSA 83476, CPATSA 79151, CPATSA 79150, CPATSA 79591, CPATSA 90194, CPATSA 79144, CPATSA 79148, CPATSA 79558 e CPATSA 79134, receberam nota média acima de 3 , nas duas avaliações realizadas pela escala de notas indicando uma moderada resistência em relação às testemunhas suscetíveis, Biloela e West Australian (Tabela 2; Figuras 3 e 4).

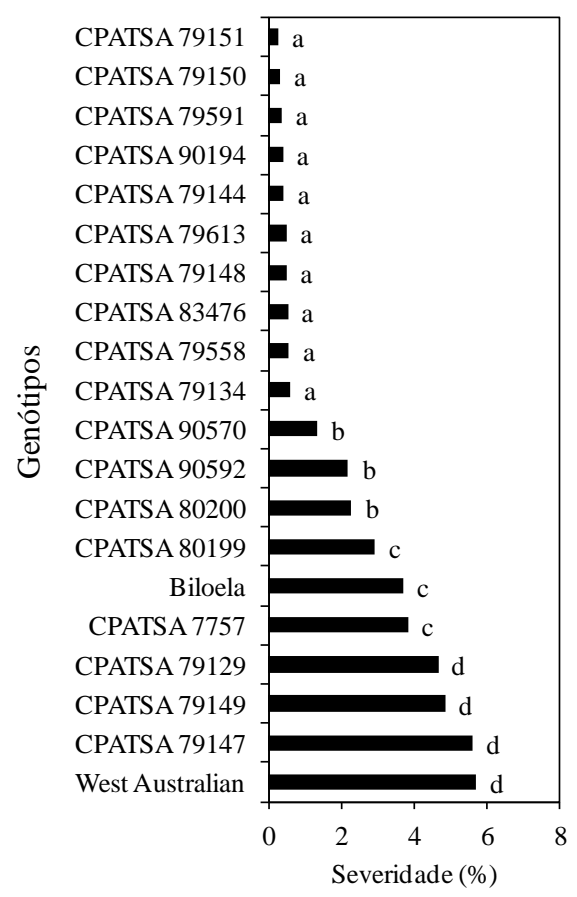


Figura 3. Reação de acessos de capim buffel ( $C$. ciliaris) à mancha foliar causada por $P$. grisea $\mathrm{em}$ campo sob infecção natural em Petrolina, Pernambuco, em março de 2020. Barras com mesma letra não diferem pelo teste de Scott \& Knott $(\mathrm{p}<0,05)$. Severidade média de 10 folhas coletadas no terço médio das plantas Fonte: Antonio et al. (2021).

Estes acessos também apresentaram uma baixa severidade de doença na parte aérea, em avaliação complementar realizada em março de 2020 em campo em relação a essas testemunhas (Figura 4).

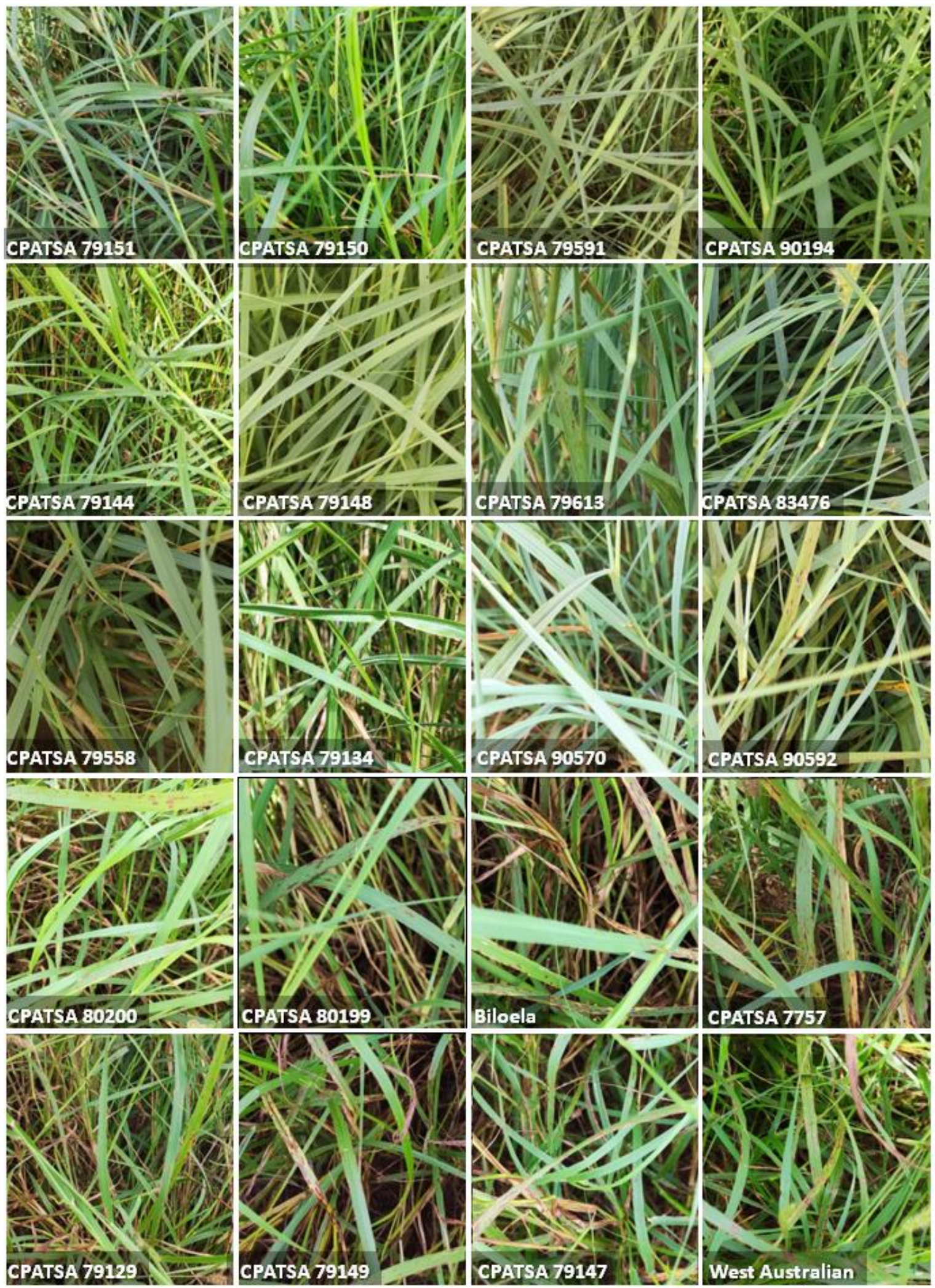

Figura 4. Reação de genótipos de capim buffel (Cenchrus ciliaris.) à mancha foliar causada por Pyricularia grisea em campo em Petrolina-PE, sob infecção natural em março de 2020. Fonte: Antonio et al. (2021). 
A cultivar de capim buffel Biloela é uma das mais difundidas e utilizadas no semiárido brasileiro assim como as cultivares Gayndah, Americano e Molopo, todas têm suscetibilidade ao fungo P. grisea (Perrott \& Chakraborty, 1999; Rodriguez et al., 1999).

Apesar da mancha foliar de $P$. grisea não ser ainda um problema para o capim buffel no Semiárido brasileiro, acreditamos que estudos neste sentido sejam de suma importância para ações futuras. Segundo Perrott \& Chakraborty (1999), no sul do Texas e norte do México, essa doença era observada por vários anos, no entanto, era confundida com sintomas de estresses à seca e, só quando tomou proporções epidêmicas, é que a causa foi definida como P. grisea. Dessa forma, uma das formas mais baratas e seguras para o controle de doenças é a seleção de materiais genéticos resistentes, que pode ser realizada de forma preventiva.

Segundo Franco, Rodríguez \& Cedillo (2007), algumas áreas produtoras de capim buffel podem sofrer epidemias devido à ausência de variabilidade genética, visto que esta gramínea se reproduz por meio da apomixia obrigatória, sem fertilização sexual; o monocultivo, grandes áreas de pastagens formadas por apenas uma cultivar, criando uma situação de vulnerabilidade genética para a presença de $P$. grisea, quando em condições climáticas favoráveis ao fungo.

Em geral, apesar do semiárido brasileiro não apresentar condições muito favoráveis à $P$. grisea em todo o ano, a busca por materiais resistentes é importante para a prevenção de futuras epidemias. Ademais, uma possível seleção/adaptação do patógeno ou mesmo, em virtude das mudanças climáticas que vem se intensificando, como a concentração de chuvas em determinadas épocas do ano que pode tornar o ambiente favorável ao patógeno, ocorrendo prejuízos aos produtores. Um exemplo disso é que nos anos 90, esta doença atingiu proporções epidêmicas no sul do Texas, EUA e norte do México em pastagens semeadas exclusivamente com a cultivar Common T-4464 (cultivar americano) (Perrott \& Chakraborty, 1999; Rodriguez et al., 1999). Flores et al. (2019) verificaram, em áreas produtoras de capim buffel no México, perdas, tanto relacionadas à produção de biomassa, quanto à produção de sementes. Os resultados mostraram que, em média, $46 \%$ a $59 \%$ das plantas de capim buffel apresentavam algum tipo de dano e as perdas decorrentes de infecção por $P$. grisea em sementes foi semelhante entre as intensidades de dano com média de $42,1 \%, 37,8 \%$ e $47,9 \%$, com dano nulo a muito leve, dano leve e moderado, respectivamente.
O programa de melhoramento de plantas adaptadas às condições semiáridas da Embrapa continua com diversos estudos com o capim buffel que visa selecionar materiais mais produtivos, nutritivos e com resistência a estresses abióticos e bióticos.

\section{Conclusão}

Há variabilidade a ser explorada dentro do Banco Ativo de Germoplasma de Cenchrus da Embrapa Semiárido quanto à reação ao fungo Pyricularia grisea. Nesse sentido, novos experimentos devem ser realizados para avaliar mais acessos, para a identificação de outras fontes de resistência.

$$
\text { Os acessos CPATSA 83476, CPATSA }
$$
79151, CPATSA 79150, CPATSA 79591, CPATSA 90194, CPATSA 79144, CPATSA 79148, CPATSA 79558 e CPATSA 79134 apresentaram resistência moderada à $P$. grisea .

As cultivares Biloela e West Australian se comportaram como suscetíveis a $P$. grisea.

\section{Agradecimentos}

Os autores agradecem a Coordenação de Aperfeiçoamento de Pessoal de Nível Superior (CAPES) e ao Conselho Nacional de Desenvolvimento Científico e Tecnológico $(\mathrm{CNPq})$ pela concessão de bolsas de pesquisa para membros da equipe.

\section{Referências}

Bedendo, I. P; Prabhu, A. S. 2016. Doenças do Arroz. In: Amorin, L.; Rezende, J. A. M.; Bergamin Filho, A.; Camargo, L. E. A. (org.). Manual de Fitopatologia: Doenças das plantas cultivadas. Vol. 2, $5^{\text {a }}$ ed. Ouro Fino: Agronômica Ceres, pp. 87-99.

Farrell, H. L.; Gornish, E. S. 2019. Pennisetum ciliare: a review of treatment efficacy, competitive traits, and restoration opportunities. Invasive Plant Sci. Manag., 12, 203-213. doi: 10.1017/inp.2019.28

Ferreira, D. F. 2011. Sisvar: a computer statistical analysis system. Ciência e Agrotecnologia, 35, 1039-1042.

Flores, F. A. I.; Rivera, M. H. M.; Medina, S. M.; Martín, F. A. I.; López, R. R. 2019. Impacto economico asociado con los daños del tizón foliar en la producción de semilla del zacate buffel en el centro de Sonora, México. Revista Mexicana de Agronegocios, 45, 313324.

Franco, A. D.; Rodríguez, A. M.; Cedillo, R. G. 2007. Tizón foliar del pasto buffel: su presencia en Tamaulipas, México. Agricultura técnica en México, 33, 285-295. 
Madhavan, S.; Malathi, S.; Rabindran, R.; Paranidharan, V.; Velazhahan, R. 2014. Genetic diversity of Magnaporthe grisea isolates from rice, finger millet, buffel grass and para grass in Tamil Nadu, India. Annals of Plant Protection Science, 22, 142-147.

Marshall, V. M.; Lewis, M. M.; Ostendorf, B. 2012. Buffel grass (Cenchrus ciliaris) as an invader and threat to biodiversity in arid environments: a review. J. Arid Environ., 78, $1-12$.

Miller, G.; Friedel, M.; Adam, P.; Chewings, V. 2010. Ecological impacts of buffel grass (Cenchrus ciliaris L.) invasion in central Australia: Does field evidence support a fireinvasion feedback? Rangeland Journal, 32, 353-365. https://doi.org/10.1071/RJ09076

Mnif, L.; Chaieb, M. 2010. Net photosynthesis and leaf water potential of buffel grass (Cenchrus ciliaris L.) accessions, growing in the arid zone of Tunisia. Journal of Biological Research-Thessaloniki, 14, 231-238.

Moreira, N. J.; Lira, M. de A.; Santos, M. V. F. dos; Araújo, G. G. L. de; Silva, C. G. 2007. Potencial de produção de capim-buffel na época seca no semi-árido Pernambucano. Revista Caatinga, 20, 22-29.
Oliveira, M. C. 2005. Capim buffel (Cenchrus ciliaris L.). In: Kiil, L. H. P.; Menezes, E. A. (org.). Espécies vegetais exóticas com potencialidades para o semiárido brasileiro. Petrolina, PE: Embrapa Semiárido; Brasília, DF: Embrapa Informação Tecnológica. pp. 129-156.

Perrott, R. F.; Chakraborty, S. 1999. Pyricularia grisea causes blight of buffel grass (Cenchrus ciliaris) in Queensland, Australia. Tropical Grasslands, 33, 201-206.

Rodriguez, O.; Gonzalez-Dominguez, J.; Krausz, J. P.; Odvody, G. N.; Wilson, J. P.; Hanna, W. W.; Levy, M. 1999. First report and epidemics of buffel grass blight caused by Pyricularia grisea in south Texas. Plant Disease, 83, 398.

Sousa, F. B.; Araujo Filho, J. A. 2007. Capim búfel (Cenchrus ciliaris L.): uma opção para ovinos e caprinos. Sobral: Embrapa Caprinos, Embrapa Caprinos. Comunicado Técnico, 75. $7 \mathrm{p}$.

Tix, D. 2000. Cenchrus ciliaris Invasion and Control in Southwestern U.S. Grasslands and Shrublands. Restoration and Reclamation Review. Student On-line Journal, 6, 1-6. 